\title{
Gene bi-targeting by viral and human miRNAs
}

\author{
Isana Veksler-Lublinsky', Yonat Shemer-Avni², Klara Kedem and Michal Ziv-Ukelson*1
}

\begin{abstract}
Background: MicroRNAs (miRNAs) are an abundant class of small noncoding RNAs (20-24 nts) that can affect gene expression by post-transcriptional regulation of mRNAs. They play important roles in several biological processes (e.g., development and cell cycle regulation). Numerous bioinformatics methods have been developed to identify the function of miRNAs by predicting their target mRNAs. Some viral organisms also encode miRNAs, a fact that contributes to the complex interactions between viruses and their hosts. A need arises to understand the functional relationship between viral and host miRNAs and their effect on viral and host genes. Our approach to meet this challenge is to identify modules where viral and host miRNAs cooperatively regulate host gene expression.

Results: We present a method to identify groups of viral and host miRNAs that cooperate in post-transcriptional gene regulation, and their target genes that are involved in similar biological processes. We call these groups (genes and miRNAs of human and viral origin) - modules. The modules are found in a new two-stage procedure, which we call bitargeting, and is presented in this paper. The stages are (i) a new and efficient target prediction, and (ii) a new method for clustering objects of three different data types. In this work we integrate multiple information sources, including miRNA-target binding information, miRNA expression profiles, and GO annotations. Our hypotheses and the methods have been tested on human and Epstein Barr virus (EBV) miRNAs and human genes, for which we found 34 modules. We provide supporting evidence from biological and medical literature for two of our modules. Our code and data are available at http://www.cs.bgu.ac.il/ vaksler/BiTargeting.htm

Conclusions: The presented algorithm, which makes use of diverse biological data, is demonstrated to be an efficient approach for finding bi-targeting modules of viral and human miRNAs. These modules can contribute to a better understanding of viral-host interactions and the role that miRNAs play in them.
\end{abstract}

\section{Background}

MicroRNAs (miRNAs) are an abundant class of small noncoding RNAs (20-24 nts) that can affect gene expression by post-transcriptional regulation of mRNAs [1]. They typically base pair with sequences in the 3' UTR of mRNAs to inhibit mRNA translation or to promote their degradation. miRNAs have been shown to play important roles in various cellular and pathogenic processes, including development, cell death, immunological response, and carcinogenesis [2,3]. Since the functional characterization of miRNAs depends heavily on identification of their specific target mRNAs, numerous bioinformatics methods have been developed for this task (see e.g., [4$11]$, for a review see [12-14]).

\footnotetext{
* Correspondence: zivukelson@gmail.com

1 Department of Computer Science, Ben-Gurion University, Beer-Sheva 84105, Israel

Full list of author information is available at the end of the article
}

In recent years, it has been shown that viruses also encode miRNAs [15,16]. Although for most of the viral miRNAs no functions have yet been described, it is conjectured that these miRNAs also take part in the complex interactions between viruses and their hosts, and several scenarios have been proposed [17] (see Figure 1, where scenario numbers are written in rhombi). In the first scenario, viral miRNAs regulate viral gene expression for maintaining, e.g., replication, latency, or evading the host-immune system. For example, miR-BART2 of EBV exhibits perfect complementarity to the 3'UTR of BALF5, which encodes the viral DNA polymerase [18], and such an interaction might be essential for maintaining EBV latency. In the second scenario, host miRNAs interact with viral RNAs, thereby inhibiting virus replication, e.g., miR-32 can limit the replication of the retrovirus primate foamy virus (PFV) in cell culture through an interaction with PFV mRNAs [19]. In the third scenario, viral miRNAs regulate host genes to induce a more favorable envi- 


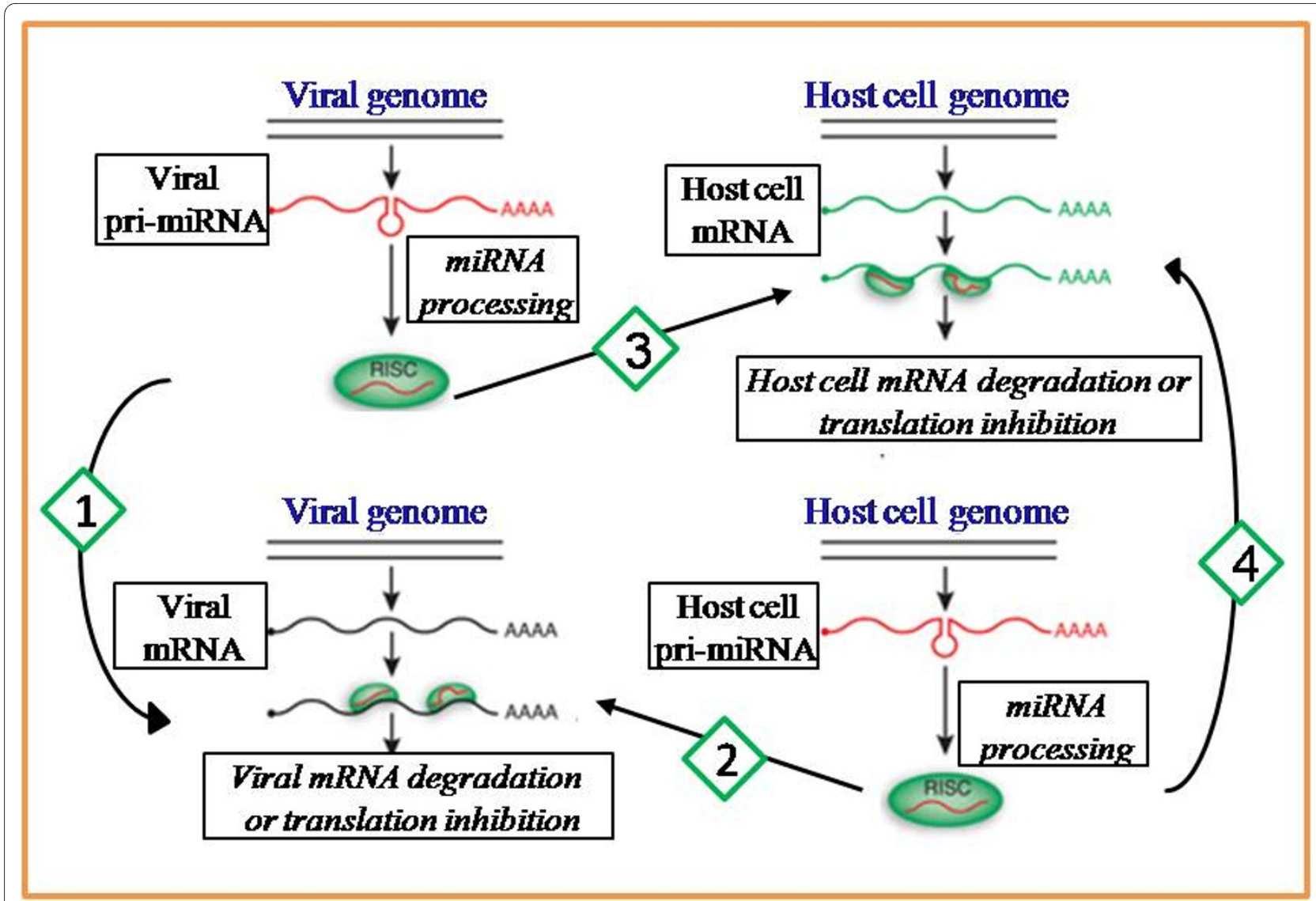

Figure 1 Four scenarios for miRNA involvement in host-virus interactions. (1) Viral miRNAs inhibit the expression of viral mRNAs. (2) Host miRNAs inhibit the expression of viral mRNAs. (3) Viral miRNAs inhibit the expression of host mRNAs. (4) Host miRNAs inhibit the expression of host mRNAs. In this case, viruses may regulate host miRNA expression and indirectly regulate host gene expression. (This figure is partially adapted from [81].)

ronment for the virus. The regulated genes are most likely related to antiviral response, apoptosis, interferon system, signal transduction, or cellular proliferation $[20,21]$. Stern-Ginossar et al. [22] showed that human cytomegalovirus (HCMV) miRNA, miR-UL112, inhibits the translation of a cellular gene MICB, which is normally activated when cells are subjected to severe stress, such as viral infection. MICB marks these cells for destruction by natural killer cells. The resulting absence of MICB protein protects $\mathrm{HCMV}$-infected cells against lysis by natural killer cells.

Viruses may affect host miRNAs for their own advantage, by regulating up or down the expression of these miRNAs. This happens in the fourth scenario reported in [23]. In their research, host miR-17 and miR-20a were found to be downregulated following human immunodeficiency virus (HIV-1) infection. These miRNAs target the 3'UTR of host gene PCAF, which has been proposed to promote HIV-1 transcriptional elongation. Hence, this downregulation is needed for efficient replication of the virus.
In our work we will examine the combination of the last two scenarios, assuming that both human and viral miRNAs play an active role in regulating genes that either promote or inhibit viral replication or life cycle.

\section{Cooperativity of miRNAs in gene regulation}

Previous studies show that one miRNA may have several target genes. Furthermore, one mRNA can be targeted by multiple miRNAs, reflecting cooperative translational control $[5,24,25]$. Experimental evidence indicates that multiple target sites (of one or more miRNAs) in the same 3'UTR can potentially increase the degree of translational repression [9]. In addition, microarray analyses [26] reveal that most of the miRNAs only modestly affect their mRNA targets. Recent findings show that some virus encoded miRNAs have sequence homology to their host human/mouse miRNAs, mainly in the seed region (see review [21]). This is one of many mechanisms that are used by viruses to exploit their host [27-30]. We were intrigued by an additional possibility in which viral and host encoded miRNAs target the same mRNA, possibly in different target sites, to promote gene silencing. It 
would make sense that viral miRNAs that share targets with human miRNAs may contribute to increasing the translational repression and tighten the regulation which already exists at a low level in the cell (by the host regulation machinery). This important question motivated us to study and predict the cooperativity of viral and host miRNAs on host genes.

In this paper, we present a new computational method for examining cooperative effects of viral and host miRNAs on the regulation of host genes. We find modules consisting of both viral and human miRNAs, and their common target genes, that are involved in similar biological processes (according to GO). The mathematical formulation of our problem is an extension of a related problem, where the sought modules consist of miRNAs and genes of the same species. Below we present some studies that worked to solve the latter problem. Yoon and De Micheli [31], the first to address that problem, represent the multiple relations between miRNAs and target genes by a weighted bipartite graph, and find bi-cliques in the graph that represent the miRNA-mRNA modules (see Figure 2). They predict modules using target prediction based on sequence information.

Two other approaches combine several information sources to extract the modules, including predictions of miRNA target genes and their respective expression profiles. Joung et al. [32] apply a genetic algorithm, based on coevolutionary learning, to find an optimal miRNAmRNA module. Tran et al. [33] use a rule-based learning method to identify the modules.

In general, finding bi-cliques in bi-partite graphs can be formulated as a bi-clustering problem (reviewed in [34]), which is known to be NP-complete. Therefore, most of the methods that address bi-clustering are based on heuristic approaches, which may miss good solutions. Alternatively, applying naive exhaustive enumeration to this

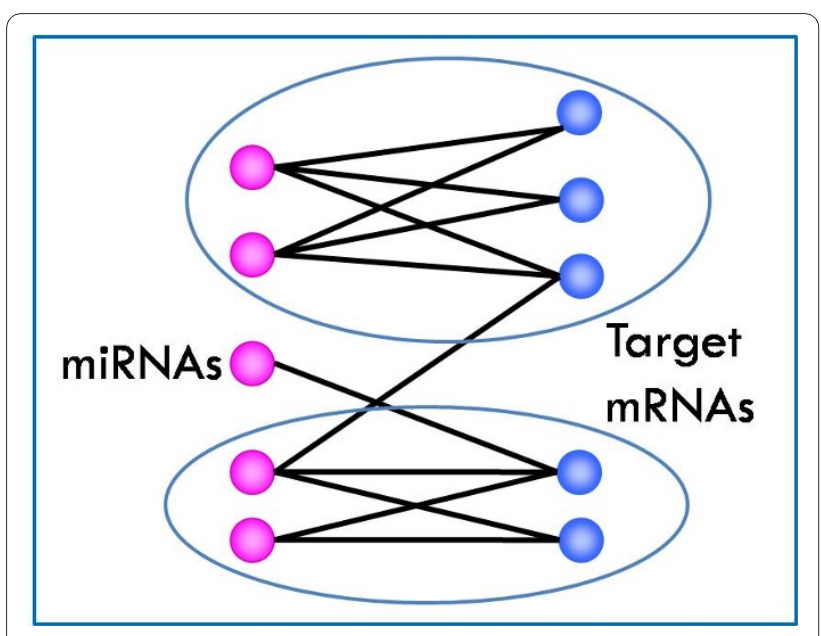

Figure 2 Bipartite graph. Bi-cliques in the graph are circled. problem is extremely time consuming to the point of being impractical. The problem becomes even more complicated when searching for cooperative human-viral miRNA modules. Directly applying one of the existing biclustering algorithms to a united set of both human and viral miRNAs may be insensitive to the contribution of viral miRNAs. This is due to the wide imbalance between the numbers of human miRNAs and viral miRNAs (hundreds of human miRNAs versus tens of viral miRNAs identified to date).

In order to be able to control the composition of the sought modules in terms of viral and human miRNA participants, a new type of clustering algorithm is needed. And indeed, in this paper we describe a bi-targeting algorithm which we developed (see Methods). We apply a branch and bound approach to develop and prune an enumeration tree of groups of human genes that are targeted by the same set of viral and human miRNAs. This algorithm is very efficient since we exploit the fact that the number of known viral miRNAs is relatively small, and that we demand a quorum on the number of miRNAs in the modules (a minimum number of viral and human miRNAs). This "pruning by quorum" allows us to apply enumeration in practical run time. We implemented our bi-targeting algorithm into a software system that combines a variety of biological data sources, such as: genomic sequences, miRNA expression measurements, GO annotations, and tissue context. The combination of several information sources in the task of module detection minimizes noise and errors accompanying each information source, resulting in more accurate results.

In addition, we developed a very flexible and efficient target prediction algorithm, which we briefly describe in the next section, and in more detail in the Appendix Section. We use it as a preprocessing step to the module search.

Using the bi-targeting system, we study the cooperative regulation of human and Epstein Barr Virus (EBV) miRNAs on human mRNAs in various lymphomas related to EBV. The significant modules are picked by a sampling procedure (described in the next section). We validate two of these modules by surveying information from the biological and biomedical literature. The rest of the significant modules can be found at http://www.cs.bgu.ac.il/ حvaksler/BiTargeting.htm.

We believe that the results of this research may have far-reaching implications, as they may be applied to the diagnosis and treatment of viral infections, as well as for a wider understanding of viral-induced diseases and the role that miRNAs plays in them. Our study can contribute to the discovery of genes that regulate virus-host interactions, especially, but not only, in chronic infections, and can serve as targets for therapy $[35,36]$. 


\section{Methods}

Our method consists of two stages: a target prediction stage and a module search stage. In the first stage, we perform all against all dynamic programming on two sets of sequences (miRNAs and mRNAs) in order to predict, for every miRNA in the set, its target mRNAs. In the second stage we look for modules that are enriched in some biological process, using the results from the first stage and information from Gene Ontology and the miRNA Atlas. We elaborate more on the second stage since this is the main goal of our paper. The interested reader may find a description of the first stage in the Appendix Section.

\section{Stage 1 - Predicting microRNA targets}

The purpose of this stage is to perform an efficient genome-wide target prediction of all the miRNAs against all the possible subsequences of a set of mRNAs. miRNA target prediction is a well-studied problem, with numerous tools and approaches available. Each of these tools accommodates a variety of principles and features of target recognition, for example typical base pairing patterns, thermodynamic analysis of the miRNA-target duplexes, conservation of the target site among related species, and accessibility of the target site. For a review see $[13,14]$.

The amount of the data we need to analyze is huge (thousands of genes and hundreds of miRNAs) and thus applying existing tools to this analysis would be too time consuming. Hence, we developed our own efficient tool for this task (which can serve as a plug-in filter to other target prediction tools). The method we propose extends the threshold all-against-all sequence alignment algorithm $[37,38]$.

We store the miRNA sequences in a prefix tree (trie) and the mRNA sequences in a separate prefix tree (for details see the Appendix Section). We then apply dynamic programming to compute hybridization error between a prefix from the mRNA tree and a prefix from the miRNA tree, for each such pair. If, in one comparison, the number of errors we encounter is larger than a given threshold, then we stop comparing this pair and its descendants in the respective trees (prune the subtrees). Those miRNA-target pairs that survived the error threshold can be further checked for duplex energy by the RNAduplex program [39]. This results in linking each miRNA in our data set to its predicted target genes.

This method is efficient since it simultaneously checks sets of mRNA sequences (and, respectively, miRNA sequences) that share a prefix in the mRNA (respectively, miRNA) prefix trees. Moreover, it goes over all the duplexes, and prunes those with a hybridization error above a threshold, allowing a very efficient filtration.

\section{Comparison with existing approaches}

We compare our target prediction method with three existing tools (those that provide downloadable code).
The tools are miRanda [5], PITA [40] and RNAhybrid [10]. Our dataset consists of 873 experimentally validated miRNA-gene pairs out of 99 human miRNAs and 640 mRNAs (from miRecords [41]). We downloaded the 3'UTR sequences of these mRNAs from Ensembl Biomart [42], a total of 2183 different transcript sequences. In our results, an miRNA-mRNA pair is considered a hit if the miRNA is found to target one of the transcripts of the mRNA. We ran our target prediction method with the constraints listed in Table 1. miRanda was run with default parameters. The parameters of PITA and RNAhybrid were set to be close to ours (seed 2-8, and the maximal bulge size, in RNAhybrid was set to 6). We compare the results of these four methods in Table 2. The information which we compare includes: the total number of predicted pairs, the number of TP predicted pairs (sensitivity) and the running time. For our target prediction tool, the total number of predicted pairs falls within the ballpark of both PITA and miRanda. RNAhybrid, on the other hand, predicts twice as many pairs. As far as sensitivity goes, our results pretty much match that of PITA and outperforms miRanda by far. RNAhybrid gave the best sensitivity, (however this comes at the cost of a very high number of total predicted pairs). When comparing running times, our tool noticeably outperformed all the other engines, this in spite of keeping up competitive sensitivity and total number of predicted pairs. Recall that fast running time was the main objective for developing our fast filtration.

\section{Stage 2 - Finding modules - the enumeration algorithm}

In this section we describe our enumeration algorithm for finding modules that are statistically enriched in biological processes (GO categories). An important objective in our study is to ensure that the modules found by our method contain miRNAs that are mutually expressed and thus can cooperatively regulate gene expression. In order to ensure this condition, we use the miRNA Atlas [43]. This Atlas provides miRNA expression profiles in

Table 1: Constraint parameters for the target prediction algorithm.

\begin{tabular}{ll}
\hline seed location & $2-8$ \\
\hline maximum GU pairs in seed & 1 \\
\hline maximum GU pairs total & 4 \\
\hline maximal number of mismatches/gaps & 6 \\
\hline maximal size of bulge in the target & 6 \\
\hline maximal size of bulge in miRNA & 6 \\
\hline
\end{tabular}


Table 2: A comparison between our method and other target prediction tools.

\begin{tabular}{llllc}
\hline Tool & $\begin{array}{l}\text { Total number of } \\
\text { predicted pairsa }\end{array}$ & $\begin{array}{l}\text { Number of TP } \\
\text { predicted pairsb }\end{array}$ & Sensitivity & Running time \\
\hline miRanda & 22,857 & 309 & $35 \%$ & 2 hours and 42 minutess \\
\hline PITA & 28,032 & 661 & $75 \%$ & 5 hours and 10 minutess \\
\hline RNA hybrid & 43,693 & 731 & $83 \%$ & $\mathbf{4 0}$ seconds \\
\hline Our method & 24,571 & 625 & $71 \%$ &
\end{tabular}

different conditions (i.e., different organ systems and cell types, of normal and malignant tissues). We focus on a set of conditions where both human and viral miRNAs (of the virus of interest) are expressed. Based on these data sources, we address the following problem.

Given a GO category $C$, a miRNA atlas condition $A$, expression level threshold $t$, a p-value threshold $p$, the minimal number of human and viral miRNAs required in a sought module $q_{1}$ and $q_{2}$ (the quorums), respectively. Find all the modules composed of miRNAs whose expression in $A$ is greater than $t$, which include at least $q_{1}$ and $q_{2}$ human and viral miRNAs, respectively, and whose intersection of the target-sets of human genes yields an enrichment $p$-value smaller than $p$ in $C$.

The enrichment is measured using a hypergeometric $\mathrm{p}$ value [44]. The hypergeometric $p$-value measures the statistical significance of the overlap between the target genes and the genes in the considered category (for more details see the Appendix Section).

For each GO category $C$ and each Atlas condition $A$ of interest, we perform the enumeration process as follows. Let $H$ and $V$ be the set of human and viral miRNAs. Let $H_{A}$ and $V_{A}$ be the subset of human and viral miRNAs expressed in $A$. Let $T_{A}$ be the set of their target genes (each gene in $T_{A}$ is targeted by at least $q_{1}$ human and $q_{2}$ viral miRNAs); and let $T C_{A}=T_{A} \cap C$ (see Figure 3).

Our enumeration algorithm dynamically constructs and prunes an enumeration tree, which is built from the genes in $T C_{A}$. A path in the tree represents a potential module. The module consists of the genes in the inner nodes of the path and a list of miRNAs (which target all these genes) in the leaf (see, e.g., Figures 4 and 5). At the end of the run of the algorithm, the enumeration tree stores in its leaves all the modules that satisfy the quorum constraints. The pseudocode of our algorithm is found in the Appendix Section. Below we supply an illustration of the algorithm applied on the data from Table 3, with the quorum constraints $q_{1}=2$ and $q_{2}=1$. We initialize the tree to consist of a root node and one dummy leaf node with all the human and viral miRNAs in $A$ (see the leftmost leaf in Figure 4(a)). Genes are inserted to the tree one by one in order of increasing number of hits of human miRNAs on the gene $(b, c, d, a)$. Each inserted gene, $g$, is connected by an edge to the root and becomes a root of newly generated copies of all its preceding siblings in the tree. The leaves of the new subtree contain intersections of $g$ 's miRNA sets (human and viral) with the corresponding sets in the siblings' leaves. When these intersections do not satisfy the quorum constraints we prune the unsatisfying edge from the tree. (See the node for gene $b$, and then for gene $c$, in Figure 4(a). The leaves of the subtree rooted at $c$ contain the intersections of the set of miRNAs hitting $c$ with the set of the dummy leaf, and of $b$. Notice that the respective intersections of the miRNA sets hitting $b$ and $c$ are empty. Thus the edge from $c$ to $b$ is pruned from the tree).

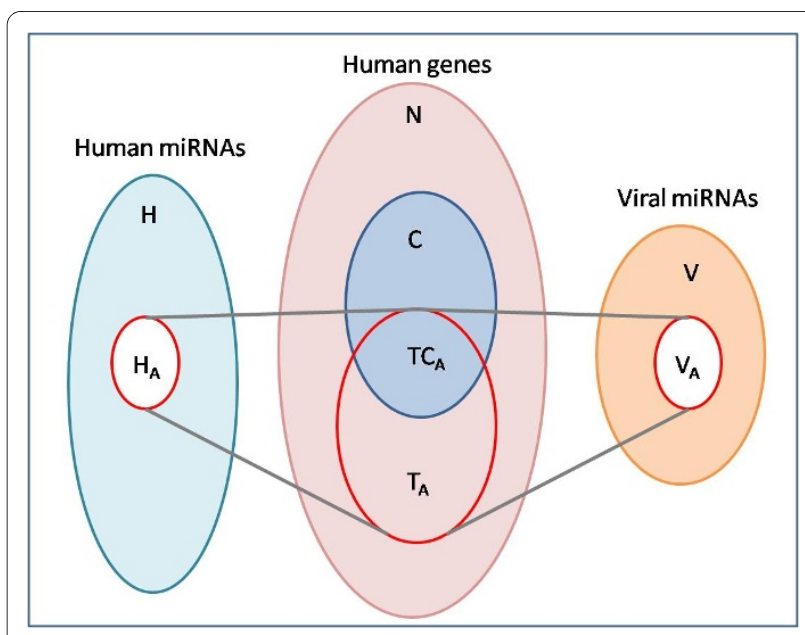

Figure 3 An illustration of the connection between Atlas condition and GO category. $H$ is all the known human miRNAs and $V$ is all known viral miRNAs. $H_{A}\left(V_{A}\right)$ are the human (viral) miRNAs expressed in Atlas category $A$. They target gene set $T_{A}$. $T C_{A}$ consists of the targets of $T_{A}$ that belong to $G O$ category $C$. 
Table 3: miRNA target information.

\begin{tabular}{|c|c|c|c|c|}
\hline $\begin{array}{l}\text { Genes } \\
\text { miRNAs }\end{array}$ & $a$ & 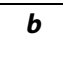 & c & $d$ \\
\hline human-miR-1 & $\sqrt{ }$ & & & $\sqrt{ }$ \\
\hline human-miR-2 & $\sqrt{ }$ & & $\sqrt{ }$ & \\
\hline human-miR-3 & & $\sqrt{ }$ & & $\sqrt{ }$ \\
\hline human-miR-4 & $\sqrt{ }$ & & $\sqrt{ }$ & \\
\hline human-miR-5 & & $\sqrt{ }$ & & $\sqrt{ }$ \\
\hline human-miR-6 & $\sqrt{ }$ & & $\sqrt{ }$ & \\
\hline viral-miR-A & $\sqrt{ }$ & & $\sqrt{ }$ & \\
\hline viral-miR-B & & $\sqrt{ }$ & & $\sqrt{ }$ \\
\hline total human miRNAs & $1,2,4,6$ & 3,5 & $2,4,6$ & $1,3,5$ \\
\hline total viral miRNAs & $A$ & B & A & B \\
\hline
\end{tabular}

In Figure 4(b) we show the tree after inserting $d$ and making it the root of all its preceding siblings: the dummy node, $\mathrm{b}$ node, and $\mathrm{c}$ node. In the leaves of the subtree rooted at $\mathrm{d}$ are the intersections of the respective miRNA sets. Now two prunings occur in the enumeration tree. The original node $b$ (in the dotted rectangle) and its targeting miRNAs, are now fully contained in the subtree rooted in $\mathrm{d}$, thus $\mathrm{b}$ is now redundant and deleted from the enumeration tree. As in 4(a), the sets of miRNAs of $c$ and of $d$ are both empty and the edge from $d$ to $c$ is pruned (solid rectangle).

In Figure 4(c) we illustrate adding $a$, making it the root of the subtrees of its siblings. Intersecting $a$ 's miRNA sets with the siblings' miRNA sets, resulting in deleting $c$ (dotted rectangle) and pruning $d$ (solid rectangle).

At the end of the process, the enumeration tree contains all the modules (see Figure 5). The leaves of the tree are traversed and their p-value is computed. The modules are then filtered (see the sampling procedure below) and reported according to ascending $\mathrm{p}$-value.

\section{The sampling procedure}

To set a cutoff p-value for significant modules, we estimate the distribution of the hyper-geometric p-value scores for every $<A, C>$ pair as follows. We sample, from the full set of miRNAs, two subsets of human and viral miRNAs, of same size as $H_{A}$ and $V_{A}$, respectively. Then we find their target genes that satisfy the quorums, and intersect them with $C$. Next, we perform the enumeration algorithm on the genes in the intersection and store the hypergeometric p-values of the obtained modules. We repeat this sampling process 10,000 times, and compute the distribution of the obtained p-values. This distribution allows us to pick a significant $\mathrm{p}$-value for our reported modules. We pick the p-value that is in the 0.01 quantile of the distribution to be the p-value cutoff for our reported modules.

\section{Results}

We applied our system to the discovery of modules of genes targeted by human miRNAs and miRNAs of the Epstein Barr Virus. The Epstein-Barr virus (EBV) is a human herpes-virus that infects over $90 \%$ of the human population worldwide. EBV can infect B lymphocytes and epithelial cells, and typically establishes long-term asymptomatic latent infection in memory B lymphocytes. Nevertheless, EBV infection has oncogenic potential, which can result in a number of malignancies, including Burkitt's lymphoma, Hodgkin's lymphoma, nasopharyn- 


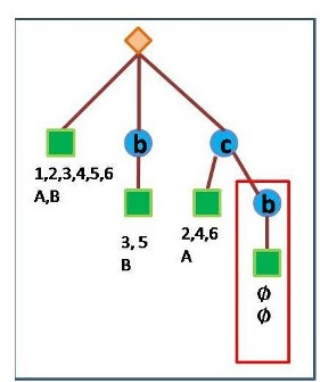

(a)

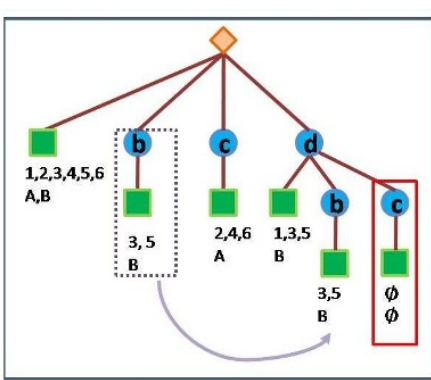

(b)

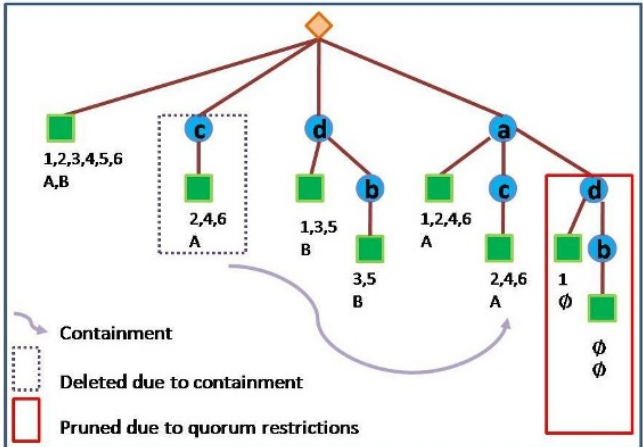

Figure 4 The process of building the enumeration tree for target information in Table 3. The rhombus represents the root of the tree. The circles represent the genes inserted into the tree. The squares represent the leaves, which store two sets of miRNAs - human and viral. These miRNAs are common "targeters" to all the genes in the path from the leaf to the root. Viral miRNAs are labeled with uppercase letters and the human miRNAs are labeled with numbers. The rectangles indicate modules that are pruned from the tree for one of two reasons: (1) modules that break the quorum are in solid rectangles (in this example the required quorum is 1 viral miRNA and 2 human miRNAs); (2) redundant modules (which are fully contained in another module) are in dotted rectangles. The arrow indicates the containment, starting from the redundant module and ending in the bigger one. The three sub-figures (a), (b), and (c) show the insertion of genes $b$ and $c, d$, and $a$ into the tree, respectively.

geal carcinoma, and lymphoproliferative diseases, especially among immunocompromised individuals [45]. During the latent infection of EBV, only a limited subset of its genes is transcribed, allowing it to evade immune recognition.

Latency gene expression is classified into three groups: type I - expressing only nuclear protein EBNA1; type II expression of EBNA1 and membrane proteins LMP-1, 2A, 2B; type III - six nuclear proteins EBNA-1, 2, 3A, 3B, $3 \mathrm{C}$, LP, and three membrane proteins LMP-1, 2A, 2B. The noncoding RNAs EBER1 and EBER2 and a set of EBV-encoded miRNAs are differentially expressed in all three forms of latency [46].

\section{Datasets}

The set of human and viral mature miRNA sequences was downloaded from the miRNA registry [47] contain-

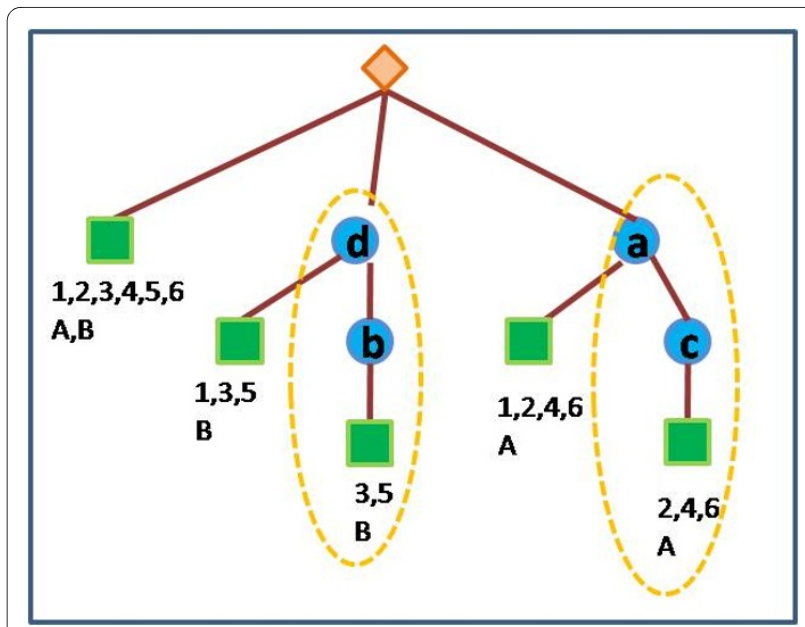

Figure $\mathbf{5}$ The final enumeration tree. The modules found by the enumeration algorithm (encircled by ellipses).

ing 866 human miRNAs and 39 EBV miRNAs (mature sequences). The set of human 3'UTR sequences was extracted from the Ensembl's Biomart [42], database Ensembl 53. Each 3'UTR in this set is annotated with an Ensembl gene and transcript ID.

The gene ontology (GO) associations and ontology structure files were downloaded from [48]. Each 3'UTR was attributed to the corresponding gene name in the ontology file, using the Ensembl gene and transcipt IDs.

The set of 3'UTRs was filtered to contain 3' UTRs from genes that are expressed in $\mathrm{B}$ cells (data taken from Ensembl Biomart) and that have at least one biological process annotation. This resulted in 7208 sequences (different transcripts) from 4773 genes. UTRs longer than $3000 \mathrm{nts}$, were shortened to $3000 \mathrm{nts}$. miRNA expressions were taken from the miRNA Atlas. Eight tissue samples from the Atlas, expressing both human and EBV miRNAs, were found to be relevant. Seventy-two GO categories (that contain 10-300 genes) related to apoptosis, immune response, signal transduction, proliferation, and cell cycle were chosen.

\section{The target prediction stage}

In the first stage we ran all-against-all target prediction between the miRNA and 3'UTR sets described above. The target prediction was carried out with the constraints listed in Table 1, which are derived from the literature (e.g. $[12,22,49])$. In addition we checked duplex energy at the leaves of the prefix tree (see Methods). The duplex energy was normalized by the energy of the duplex formed between the miRNA and its complement, and if the resulting ratio exceeded a threshold of 0.4 the target prediction was accepted as positive.

Running the target prediction on 866 human miRNAs (39 EBV miRNAs) and 7208 human mRNAs (3' UTRs) 
resulted in 283362 human miRNA/human mRNAs (11663 EBV miRNA/human mRNAs) pairs. We analyzed some characteristics of the putative target-binding association (for the human miRNA-mRNA pairs). Figure 6(a) shows the distribution of the number of target mRNAs per each human miRNA, and Figure 6(b) shows distribution of the number of human miRNAs targeting each mRNA. According to this analysis, one human miRNA binds putatively to the 3 'UTR of 327 mRNAs on average (4.5\% of the total number of mRNAs in the dataset). Alternatively, one mRNA is targeted by 39 human miRNAs on average (4.5\% of the total number of human miRNAs in the dataset). An analysis of the efficiency of our filter can be found in the Appendix Section.

\section{The predicted modules and supporting evidence}

In this stage we used the target prediction results from the previous stage. The enumeration process was carried out on $72 \mathrm{GO}$ categories versus 8 Atlas conditions, resulting in $576<A, C>$ pairs, where $A$ denotes an Atlas condition and $C$ denotes a GO category. We found 54 modules that fulfilled the following quorum constraints, $q_{1}=2, q_{2}$ $=1$, and had a $\mathrm{p}$-value lower than the cutoff (obtained by a sampling procedure for each such pair. See Methods for details). Modules with fewer than two genes were filtered out. The expression level threshold $t$ was calculated for each miRNA as the average expression among all the Atlas conditions related to $B$ cells. Among the GO categories tested in our experiment, there are dependent categories that contain the same genes. Among Atlas conditions sometimes groups of expressed miRNAs overlap. In order to reduce the redundancy of the reported modules we combined identical modules from different GO categories and Atlas conditions. This procedure resulted in 34 modules. Below we present and analyze two selected modules that were found by our method. The full set of potential modules can be obtained at http:/ /www.cs.bgu.ac.il/ vaksler/BiTargeting.htm.

Module I( see Figure 7(a)) was obtained from genes related to "induction of apoptosis" and miRNAs that are expressed in Burkitt lymphoma. The module consists of three human miRNAs, miR-17, miR-20a, and miR-15b, one viral miRNA, miR-BART5, and three human genes. A thorough literature search supported our predictions as follows. miR-17 and miR-20a belong to the genomic cluster miR-17-92. The cluster consists of six miRNAs that are tightly grouped within an 800 base-pair region of human chromosome 13 [50]. The miR-17-92 cluster first attracted attention following a series of observations linking these miRNAs to cancer pathogenesis, as it undergoes amplification in several types of lymphoma and solid tumors. It was also shown that these miRNAs are tightly linked to the functions of the E2F family of transcription factors, which are critical regulators of the cell cycle and apoptosis. Furthermore, O'Donnell et al. [51] validated that E2F1 is a target of miR-17 and miR-20a. Therefore, these miRNAs lead to decreased E2F1 protein and thus attenuate E2F induced apoptosis (see Figure 8).

The third human miRNA is miR-15b. This miRNA was recently shown to regulate cell cycle progression in glioma cells by targeting cell cycle-related genes, such as CCNE1 [52]. In addition, Cimmino et al. [53] reported that its analog miR-15a (along with miR-16-1) negatively regulates BCL2, which is an anti-apoptotic gene that is often overexpressed in many types of human cancers. The participation of this miRNA in this module suggests that this miRNA may also have oncogenic roles in this particular type of malignancy, by targeting genes that promote apoptosis.

The viral miRNA miR-BART5 was shown to target a human protein PUMA [54]. PUMA plays a role in apoptosis as a direct downstream target of p53, in addition to being able to initiate apoptosis via a p53-independent mechanism [55]. Down-regulation of PUMA by miRBART5 may protect EBV-infected cells from virusinduced apoptosis. Furthermore, searching for targets of BART5 on the viral genome revealed 8 hits on the EBNALP protein. EBNA-LP promotes the expression of viral genes that expose the virus to the immune system.

Three human genes that were found to participate in this module are strongly related to apoptosis. PPP1R13B (also known as ASPP1) has an important function in cellular apoptotic pathways [56,57]. ASPP1 and ASPP2 (another member of ASPP family) are induced by the E2F1 transcription factor and cooperate with the tumor suppressor p53 and its family members p63 and p73 in trans-activating pro-apoptotic genes such as the BCL2family member BAX, the BCL2-binding component PUMA, and p53-induced gene 3 (PIG3) (see Figure 8). Inhibition of endogenous ASPP function suppresses the apoptotic function of endogenous p53 in response to apoptotic stimuli [57].

The second gene in the module is BCL2L11(also known as BIM), which is a pro-apoptotic gene that regulates cell death in mature B cells. It is thought to initiate apoptosis by binding to and inactivating pro-survival BCL2-family members, such as BCL2 [58]. Deletions or methylation of the BIM locus are found in various human B lymphomas $[59,60]$. Furthermore, it was shown previously that latent infection of Burkitt Lymphoma cells with EBV results in a significant reduction in the expression of BIM [61]. A number of recent works ([62-64]) have also identified BIM as a direct target of multiple members of the miR17-92 cluster (including mir-17 and miR-20a).

The third gene, TP63 (also known as p63), an important epithelial developmental gene, is a member of the p53 tumor-suppressor gene family. The p63 gene has two promoters, resulting in two different types of proteins 


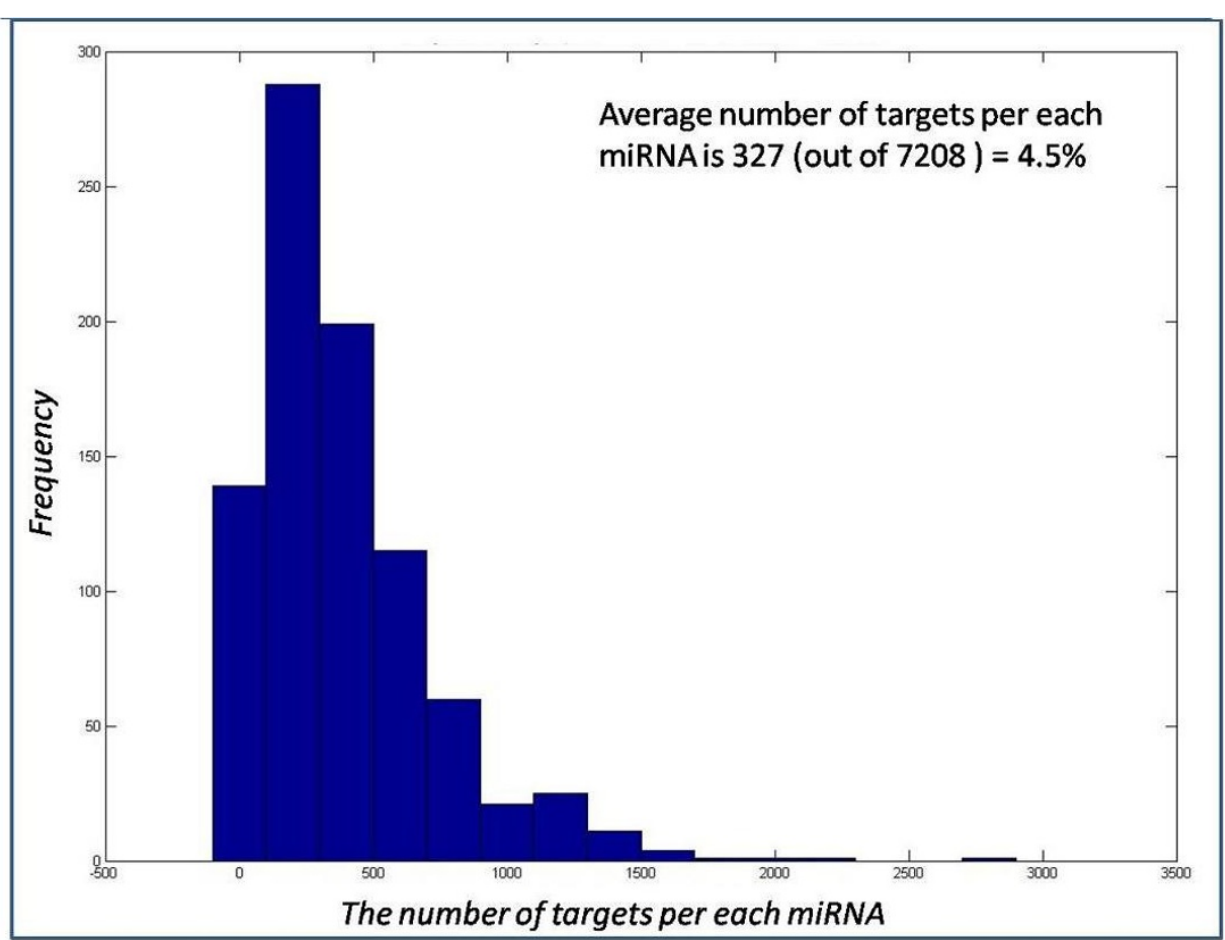

(a)

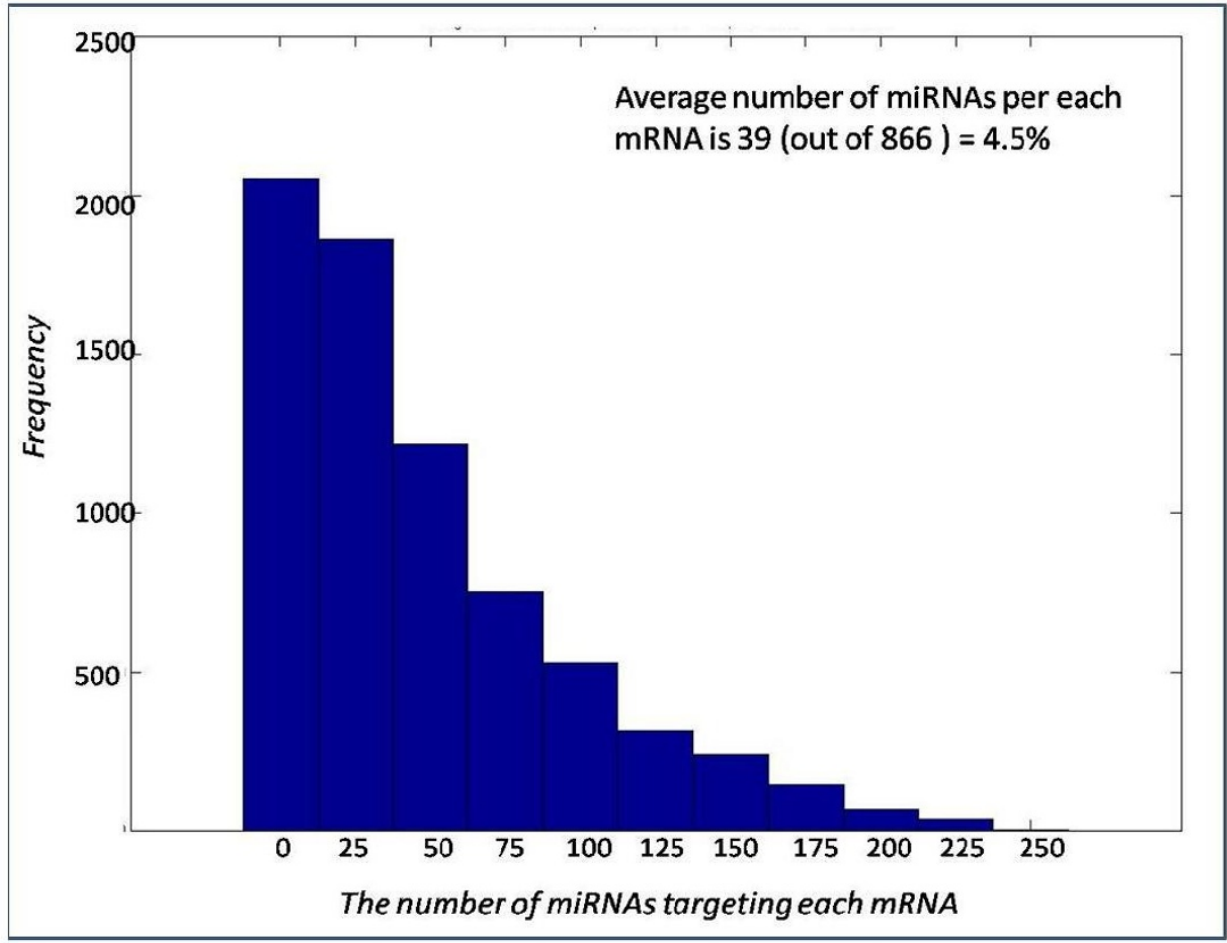

(b)

Figure 6 Characteristics of the human miRNAs/human mRNAs relationships. (a) Distribution of the number of target mRNAs per each miRNA. (b) Distribution of the number of miRNAs targeting each mRNA. 


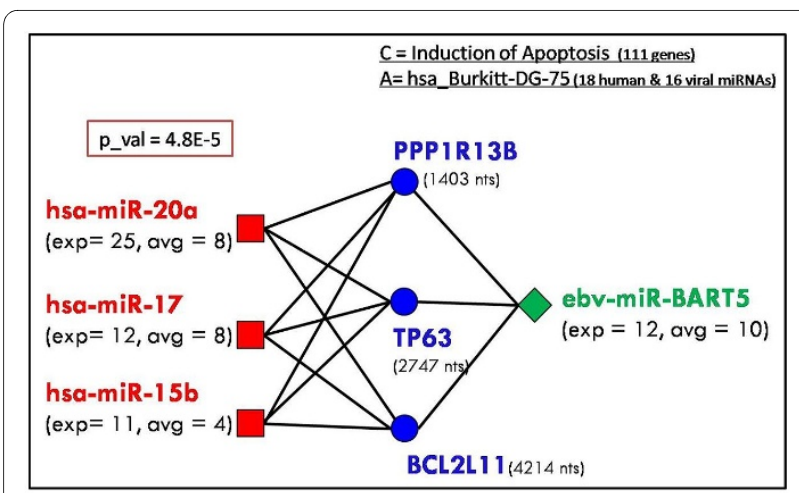

(a)

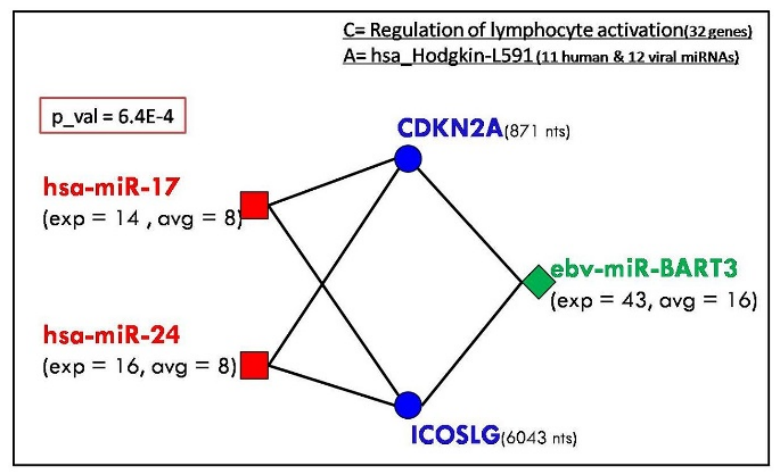

(b)

Figure 7 Two predicted modules. The length of the UTR is indicated in the parentheses near the gene name. The expression in the Atlas condition and the average expression among $B$ cell conditions is indicated in the parentheses under the miRNA name. The Atlas conditions: (a) has_Burkitt-DG-75: Burkitt lymphoma cell line established from a 10-year-old boy, EBV+; (b) hsa_Hodgkin-L591: Hodgkin Lymphoma cell line, EBV+.

with opposing functions, a p53-like protein containing the TA domain (TAp63) and inhibitory proteins lacking TA, called DNp63. TAp63 upregulates expression of proapoptotic Bcl-2 family members such as Bax and BCL2L11 [65]. p63 is capable of binding a series of p53responsive promoters and can transactivate many p53 target genes. p63 activity is regulated by proteins such as ASPP1 and ASPP2 (see Figure 8), which also modulate p53 activity. p63 can mediate apoptosis in a manner similar to that of $\mathrm{p} 53$, and it was proposed that $\mathrm{p} 63$ is essential for p53-mediated apoptosis induced by DNA damage [66]. The cooperative regulation of human and viral miRNAs of the apoptotic pathway is demonstrated in Figure 8. The dashed arrows indicate validated targets of miR17, mir-20a, and miR-BART5, and the solid arrows indicate the new regulation pattern discovered in our module. Our predictions may imply that miRNAs in this module target genes along the cascade that leads to apoptosis. In particular, these results predict that a primary function of miR-BART- 5 may be to prevent p53 mediated apoptosis by targeting different mRNA transcripts.

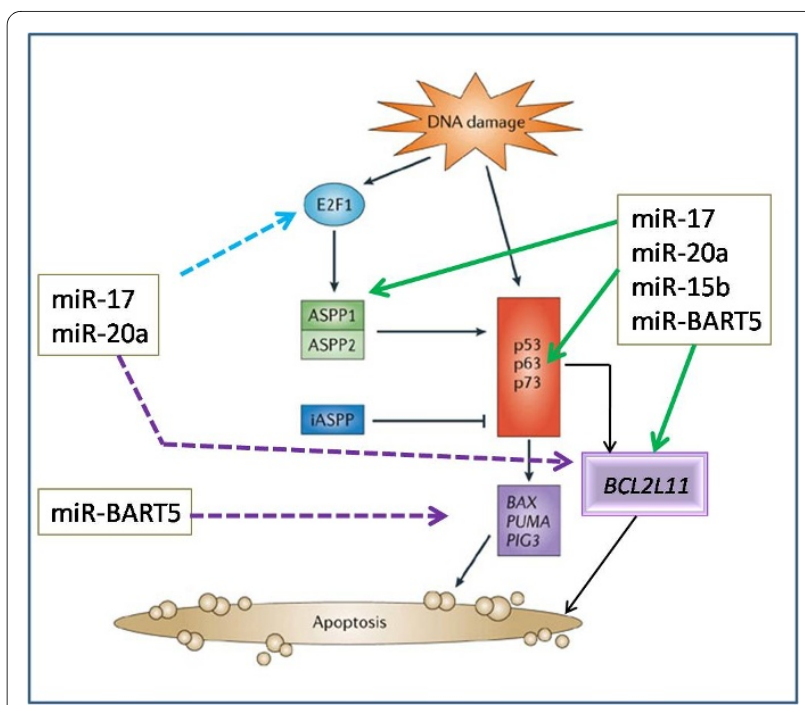

Figure 8 The function of Module I genes in cellular apoptotic pathways, and the cooperative role of human and viral miRNAs in this cascade. The dashed arrows indicate validated targets of the miRNAs, the solid arrows indicate the new regulation pattern discovered in our module (the figure is partially adopted from [56]).

Module II (see Figure 7(b)) consists of miRNAs from the Hodgkin Lymphoma cell line, hsa-miR-17, hsa-miR24, and ebv-miR-BART3, and genes from the "regulation of lymphocyte activation" category - CDKN2A and ICOSLG.

CDKN2A (also known as p16-INK4A) is a tumor suppressor that binds to the complex of cyclin D1 and cyclindependent kinase 4 to repress its ability to phosphorylate the retinoblastoma protein, and consequently, blocks cell cycle progression from $\mathrm{G} 1$ to $\mathrm{S}[67,68]$. Inactivation of this gene has been shown in a wide variety of human cancers as a consequence of mutation, homozygous mutation, or promoter methylation [69-71]. In addition, it was shown that EBV oncoprotein LMP1 blocks the expression of CDKN2A, by promoting the CRM1-dependent nuclear export of Ets2, which is an important transcription factor for CDKN2A, thereby reducing the level of its expression [72]. Furthermore, human miRNA miR-24, which presents in this module, has been demonstrated to promote cell growth through repression of this gene [73]. miR-17, in addition to its function in attenuating E2F induced apoptosis (see module I), was shown to target CDKN1A (also known as p21), a gene that functions as a regulator of cell cycle progression at G1 [74].

The second gene found in module II is ICOSLG. This gene is expressed on monocytes, dendritic cells, and $\mathrm{B}$ cells and can be induced by inflammatory stimuli in peripheral tissue. Binding to ICOSL delivers a co-stimulatory signal for $\mathrm{T}$ cell proliferation and cytokine secretion $[75,76]$. Furthermore, this gene was shown to be important in several immune responses against pathogenic 
microorganisms such as bacteria, parasites and viruses [77-79].

The two modules presented have a tight connection to the function of the EBV, which establishes a long-term latent infection in the host cells. Thus, it is reasonable to assume that EBV will use its and the host's machinery, including miRNAs, for downregulating genes that lead to apoptosis or immune response. These modules supply evidence of the cooperation between human and EBV miRNAs in the task of preventing apoptosis, promoting cell growth, and evading the immune response. It is important to note that the target sites of the human and viral miRNAs on the UTRs of the genes in this module are different. Thus this cooperation, with multiple target sites, can lead to an increased degree of translational repression.

\section{Discussion and Conclusions}

miRNAs represent a class of molecules produced by both viruses and their hosts that can benefit either the virus or the host, depending on the particular interaction. Viral miRNAs were discovered only recently, and functional relationships between viruses and viral or host miRNAs are only now beginning to be elucidated. A comprehensive understanding of the entire landscape of the miRNAmediated host-virus interactions may uncover novel pathways that promote or limit virus replication. In turn, this knowledge may lead towards the development of effective antiviral therapy and could help guide drug design.

In this work we focused on the contribution of viral and host miRNAs in regulating host genes, and thus in promoting or inhibiting viral replication or life cycle. Our method searches for modules of miRNAs (host and viral) and their common host target genes that are involved in similar biological processes.

Our method is related to bi-clustering methods that have been used in various biological issues [34]. The biclustering approach groups rows and columns simultaneously in a two-dimensional data matrix. Alternatively, a data matrix can be viewed as a bipartite graph. Previous works, dealing with module search, represent one set of nodes as miRNAs and the second set as target mRNAs; the edges represent target relations. Since in our problem, the miRNAs are of two different sources (viral and human), we cannot use the existing methods of bi-clustering. Instead, our graph can be viewed as a two-sided bi-partite graph, and the goal is to find two sided bicliques.

In order to achieve this goal, we developed a new bitargeting algorithm. The algorithm constructs an enumeration tree of human genes that are targeted by the same sets of miRNAs, and contains the modules at the end of its construction. Due to the application of a branch and bound technique, the algorithm is very efficient and enables, within a practical time, enumeration of all possible modules.

Our method combines a variety of biological data sources. Genomic sequences were used for the target prediction task. Tissue context information was used to narrow the list of human genes to genes that are expressed in the cells infected by the virus of interest (e.g. B cells in the case of EBV). miRNA expression profiles (from the miRNA atlas) and GO annotations enabled us to find potential modules consisting of miRNAs co-expressed in an Atlas condition (and have a potential to regulate cooperatively a group of genes) and genes with similar biological function from a GO category. The combination of several information sources in the task of module detection minimizes noise and errors accompanying each information source, resulting in more reliable results.

In addition, we developed our own method for target prediction, which extends the threshold all-against-all algorithm. The method performs an efficient genomewide target prediction of all the miRNAs against all the possible subsequences of a set of mRNAs. Our algorithm was able to prune a large portion of the aligned trees by utilizing the fact that the number of errors in the miRNAmRNA duplex is bounded by some threshold.

Note that the two methods we present in this paper are independent. One can use our target prediction algorithm as an efficient stand-alone target prediction system. In addition, our module finding method can use any source of target information, produced by any of the existing target prediction tools, using different principles of target recognition. The method can also be applied to other viruses, as long as their miRNA expression profiles are available (along with human miRNAs).

We applied our method to the discovery of modules consisting of human and viral (EBV) miRNAs and human genes. The identified modules display meaningful discoveries supported by the literature. Two of the modules are analyzed in detail in this paper.

Since not much is known about the function of viral miRNAs, finding modules that link the viral miRNAs and the human miRNAs, might help in understanding the role of viral miRNAs in viral infections. Thus the method developed in this work can be of help to better understand viral-induced diseases and the role that miRNAs plays in them.

\section{Appendix}

Predicting miRNA targets: a filter based on error bounded complementarity

We perform a genome-wide target prediction of all the miRNAs against all the possible subsequences of a set of mRNAs in an efficient way. The method we propose extends the threshold all-against-all alignment algorithm 
$[37,38]$. All-against-all alignment problems can be solved using dynamic programming and suffix trees [37]. Sagot used a variant of this problem to extract approximate repeated motifs from a sequence or common motifs from a set of sequences [38]. In this work we extend this idea to the task of target prediction by using prefix trees (tries).

The trie data structure [80], $\tau$, allows representation of the miRNA/mRNA sequences in an efficient way. The tree $\tau$ has the following properties: each edge of $\tau$ represents a character $c \in\{A, C, G, T / U\}$, the characters represented by sibling edges are distinct, and the concatenation of the labels of the edges on a path from the root to a leaf represents the full string inserted in the tree.

For our target prediction system we construct two tries, one for the miRNA sequences and one for sequence segments of the (reversed) mRNAs. Next, we apply the branch and bound algorithm to find complementarities between sequences from the miRNA trie and the mRNA tries. In more detail, construction of the tries is as follows.

Let $M$ be a set of $m$ miRNAs, and let $G$ denote a set of $n$ mRNA sequences in the database for the organism under consideration. Consider the trees in Figure 9, $\tau_{M}$ and $\tau_{G}$, which represent the miRNA and mRNA sequences, respectively. $\tau_{M}$ is built from the miRNA sequences in $5^{\prime} \mathrm{T}$ $3^{\prime}$ orientation. A node $u$ in $\tau_{M}$ keeps the character of the incoming edge (the empty character in the root). A leaf node, which corresponds to an miRNA, keeps in addition a boolean vector $V(u)$ initialized with $F$ (for false). Each $V$ $(u)$ has $n$ cells, one for each mRNA, and each mRNA has a running number. Cell $i$ in $V(u)$ indicates whether the $i$ 'th mRNA is the miRNA's predicted target $(V(u)[i]=T)$.

$\tau_{G}$ is built from mRNA subsequences, which are produced by partitioning the mRNA reversed ( $3^{\prime} \mathrm{T}$ 5) sequences into given size consecutive overlapping windows with a slide of 1-nt. The partitioning of the mRNA into windows ensures that every nucleotide in the mRNA will be examined as a potential starting point of a target site to one or more miRNAs. Since the mRNA sequence may have bulges when hybridized to an miRNA, we set the size of the window to be longer than the typical miRNA length, namely to 30 nucleotides. Each node in $\tau_{G}$ keeps the same data as $\tau_{M}$. It also has a list of all mRNAs for which the path from the root of $\tau_{G}$ to this node spells a substring of this mRNA (see Figure 9(b)).

Our goal now is to measure the complementarity between all the miRNAs in $\tau_{M}$ and the mRNA subsequences in $\tau_{G}$. In order to get an admissible branch-andbound search, we use a target prediction score which is monotonic with respect to the increasing length of the prefixes of the compared miRNAs and mRNAs (see Fig- ure 10). The score, which is denoted by $\min _{-} \operatorname{err}(u, v)$ is computed using dynamic programming.

\section{The dynamic programming table DP}

for any pair of nodes $(u, v), u \in \tau_{M}$, and $v \in \tau_{G}, D P[u, v]$ holds the target prediction score between the prefix of the (miRNA) sequence spelled by the path from the root of $\tau_{M}$ to node u versus the prefix of the (mRNA) sequence spelled by the path from the root of $\tau_{G}$ to node $v$, respectively.

Let $u^{\prime}$ be the parent of node $u$ in $\tau_{M}$ and let $\alpha$ be the character labeling the edge between them. Similarly, let $v^{\prime}$ be the parent of $v$ in $\tau_{G}$ and let $\beta$ be the character labeling the edge between them. If we traverse the trees in preorder traversal, then $\left(u^{\prime}, v^{\prime}\right),\left(u^{\prime}, v\right)$, and $\left(u, v^{\prime}\right)$ are computed before $(u, v)$.

The target prediction score of $(u, v)$ is computed using the recurrence formula given below:

$m i n_{-} e r r(u, v)=m i n\left\{\begin{array}{l}m i n_{-} e r r\left(u^{\prime}, v\right)+m i R N A_{-} g a p \\ \min \_r r\left(u, v^{\prime}\right)+m R N A_{-} g a p \\ m_{-} e r r\left(u^{\prime}, v^{\prime}\right)+\operatorname{match}(a, b)\end{array}\right.$

where miRNA_gap and mRNA_gap are penalties for inserting a gap on the miRNA side of the duplex or on the mRNA side of the duplex, respectively, and $\operatorname{match}(\alpha, \beta)$ is the dis-complementarity penalty between the characters $\alpha$ and $\beta$ (equal to 0 if $\alpha$ and $\beta$ are complementary, and a positive score otherwise).

Note that mismatches and gaps increase min_err. Using the restrictions on miRNA target detection based on sequence complementarity, we can bound min_err with a threshold $k$. Thus, an mRNA sequence $T$ has a chance of being a target of miRNA sequence $P$ if min_err of $P$ and $T$ is at most $k$.

\section{Observation}

The score min_err $(u, v)$ is monotone with respect to an increase in the prefix length of $P$ and $T$ (see $k$ and $k^{\prime}$ in Figure 10).

Several features that are related to the hybridization pattern between the miRNA and its targets are incorporated into this algorithm. There is an option (a) to require a seed of a given length and position (perfect complementarity region) in the hybridization between the miRNA and the mRNA, (b) to restrict the number of $G U$ pairs that are formed in the duplex, and (c) to restrict the size of each bulge formed in the duplex. The thresholds that we use for these features in our work are derived from the literature describing experimentally verified miRNA targets (see the Results Section).

The computation of min_err $(u, v)$ is done in pre-order traversal of the trees. Pruning is performed while traversing the mRNA trie. The calculation is stopped at each 


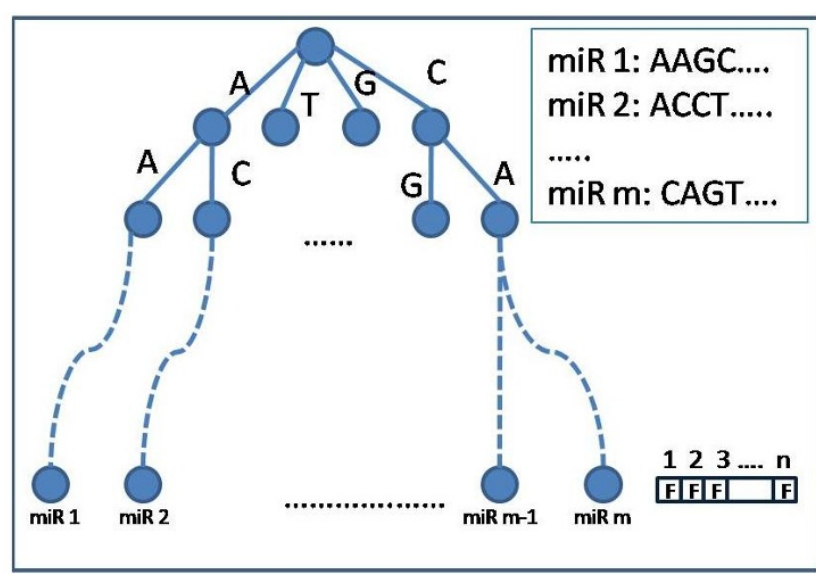

(a)

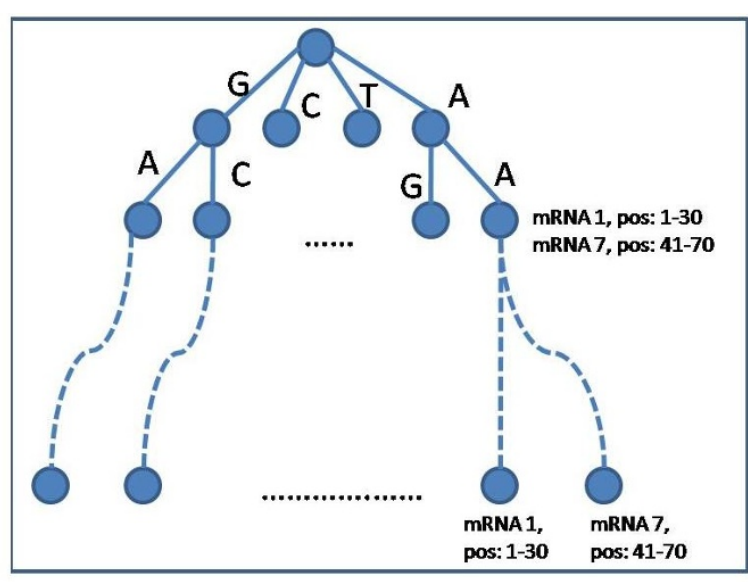

(b)

Figure 9 The prefix tries. (a) $\tau_{M}$ and (b) $\tau_{G^{\prime}}$ miRNA and mRNA tries, respectively.

node $v$ of $\tau_{G}$ where min_err $(u, v)>k$, or one of the above listed restrictions is violated. When the algorithm reaches a leaf $u$ in $\tau_{M}$, it updates $V(u)$ according to the mRNA sequences that are located in the non-pruned nodes of $\tau_{G}$. At this point a hybridization structure between the miRNA represented by leaf $u$ and the corresponding mRNA target sequences is calculated using the RNAduplex program [39]. Duplexes with a normalized free energy score (i.e., the duplex energy normalized by the energy score of the miRNA bound to its perfect complement) less than a given user threshold are filtered. After performing the DP, we are left with an miRNA tree where every leaf node $v$ is associated with the set of mRNAs which are its predicted targets, as maintained in the $T(v)$ vector.

We note that the filter described in this section is stand-alone, and can be used independently on other species with different parameters.

Filter efficiency measurements

We demonstrate the efficiency of applying our filter on the dataset, described in the Datasets subsection, with three sets of parameters as detailed in Table 4. Each UTR was partitioned into sliding windows of size $30 \mathrm{nts}$, with a

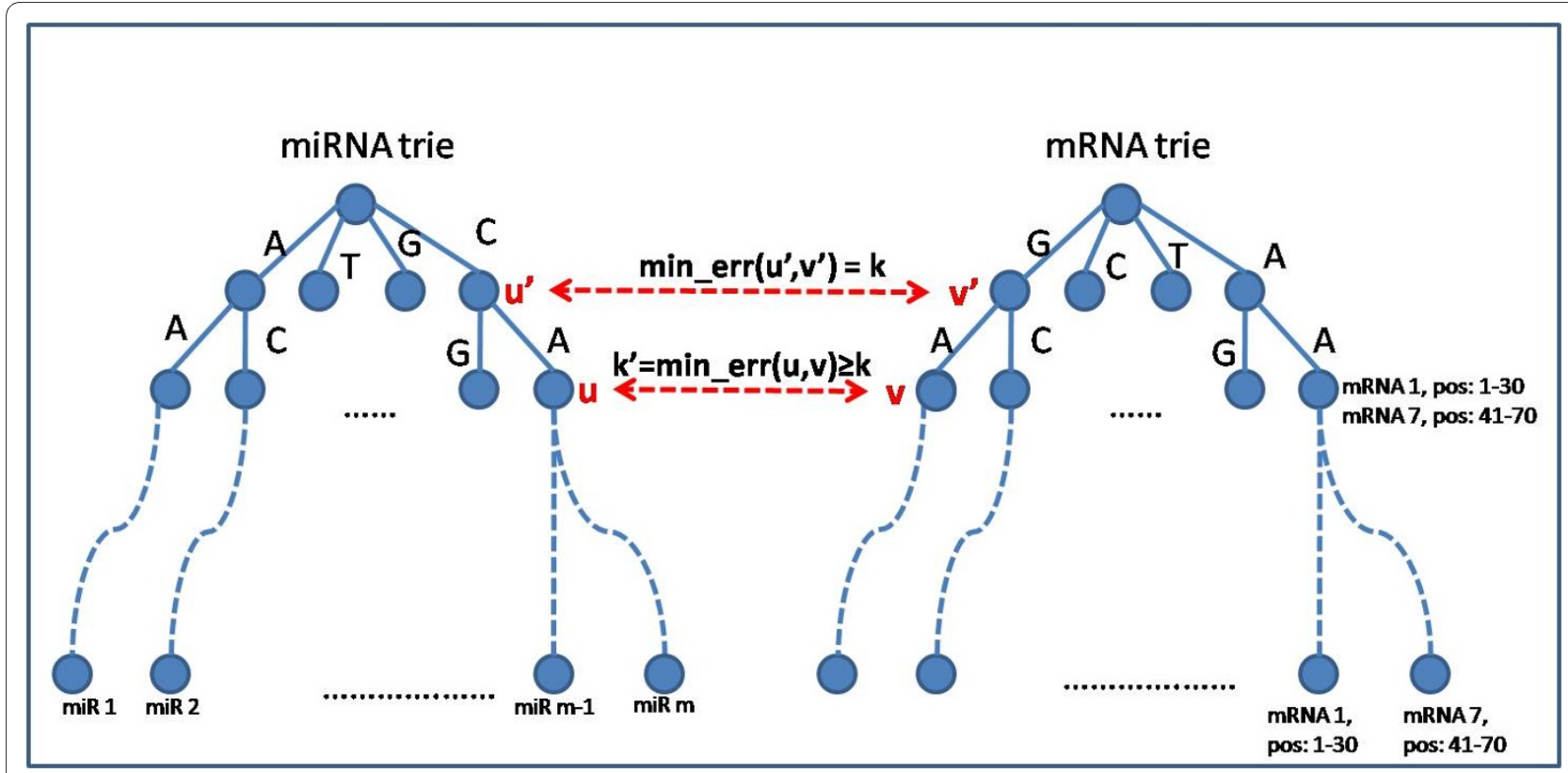

Figure 10 min_err monotonicity property. The score min_err $(u, v)$ is monotone with respect to the increase in the prefix length of the miRNA and mRNA sequences. 
sliding offset of 1 nts between frames, resulting in 7356844 subsequences. Since the RNAduplex procedure is by far the most time consuming component of our method, we measured the efficiency of our filter in terms of the number of calls to RNAduplex.

We define by the naive method a method that calls RNAduplex when there is a seed complementarity, under the constraints of seed location and number of GU pairs in the seed (see columns 1 and 2 in Table 4). Columns (A) and (B) contain the number of RNAduplex calls for the naive method and for our method, respectively, and column $(C)$ contains the percent of calls saved by our method.

In all three data sets there is a great reduction in the number of RNAduplex calls. The middle row demonstrates a much bigger save in number of calls by our method. This is mainly because of the strong constraint applied on the number of allowed errors (column 6). The reduction in the number of calls to RNAduplex is due to the compression of the sequences of both miRNAs and mRNAs into prefix trees and the strong pruning that we apply during the calculation of the hybridization score, accompanied with the constraints that we apply on potential duplexes.

\section{Hypergeometric $p$-value}

For a given module obtained for a pair $\langle A, C\rangle$, the hypergeometric p-value is computed as follows: Let $n$ be the total number of genes in the dataset and $c$ the number of genes that belong to the GO category $C$ (denoted as $N$ and $C$ in Figure 3, respectively). Let $t$ be the number of mutual targets of the miRNAs in the module and $t c$ the number of genes among them that also belong to $C$ (the latter is the result of the enumeration algorithm). The hypergeometric $p$-value of this module is:

$$
\text { pval }_{\text {hyper }}(t c \mid c, t, n)=\sum_{i=t c}^{\min (t, c)} \frac{\left(\begin{array}{l}
t \\
i
\end{array}\right)\left(\begin{array}{l}
n-t \\
c-i
\end{array}\right)}{\left(\begin{array}{l}
n \\
c
\end{array}\right)}
$$

\section{Pseudocode of the enumeration algorithm}

input : Genes - list of genes from a certain GO category. Every gene $x$ in this list has a set of human and viral miRNAs targeting it, denoted as $x . h \_m i R s$ and $x . v_{-} m i R s$, respectively.

input : $h \_m i R s, v_{-} m i R s$ - full list of human and viral miRNAs.

output: Enumeration tree

root $\leftarrow$ new Node();

dummy $\leftarrow$ new Node();

dummy.h_miRs $\leftarrow$ h_miRs;

dummy.v_miRs $\leftarrow v$ miRs;

root.children.add(dummy);

foreach gene $\in$ Genes do

node $\leftarrow$ appendSiblings(gene);

root.children.add(node);

end

Algorithm 1: construct tree

input : gene - a new gene to be inserted into the tree

input : root - the root node of the enumeration tree

input : $q_{1}, q_{2}$ - quorum restriction on human and viral miRNAs

output: Creates a new node and appends to it (as its

children) all its siblings

node = new Node(gene);

foreach child $\in$ root:children do

sibling $\leftarrow$ copy(child);

foreach leaf $\in$ sibling:leaves do

size $1 \leftarrow$ leaf.h_miRs.size;

Table 4: The efficiency of the filter on three different sets of parameters.

\begin{tabular}{|c|c|c|c|c|c|c|c|c|}
\hline \multicolumn{6}{|c|}{ Constraints } & \multirow{3}{*}{$\begin{array}{l}\text { (A) RNAduplex calls } \\
\text { in the naive method }\end{array}$} & \multirow{3}{*}{$\begin{array}{l}\text { (B) RNAduplex calls } \\
\text { by our method }\end{array}$} & \multirow{3}{*}{$\begin{array}{l}\text { (C)\% of } \\
\text { saved calls }\end{array}$} \\
\hline \multirow{2}{*}{$\begin{array}{c}\text { Seed } \\
\text { location }\end{array}$} & \multicolumn{2}{|c|}{ No. of GU pairs } & \multicolumn{2}{|c|}{ Maximal bulge size } & \multirow[b]{2}{*}{$\begin{array}{c}\text { Maximal no. } \\
\text { of errors }\end{array}$} & & & \\
\hline & in seed & total & on miRNA & on mRNA & & & & \\
\hline $2-8$ & 1 & 4 & 6 & 6 & 6 & 1819306 & 1108226 & $39 \%$ \\
\hline $2-8$ & 1 & 2 & 6 & 6 & 3 & 1819306 & 140920 & $92 \%$ \\
\hline $2-7$ & 0 & 4 & 6 & 6 & 6 & 480099 & 267087 & $45 \%$ \\
\hline
\end{tabular}




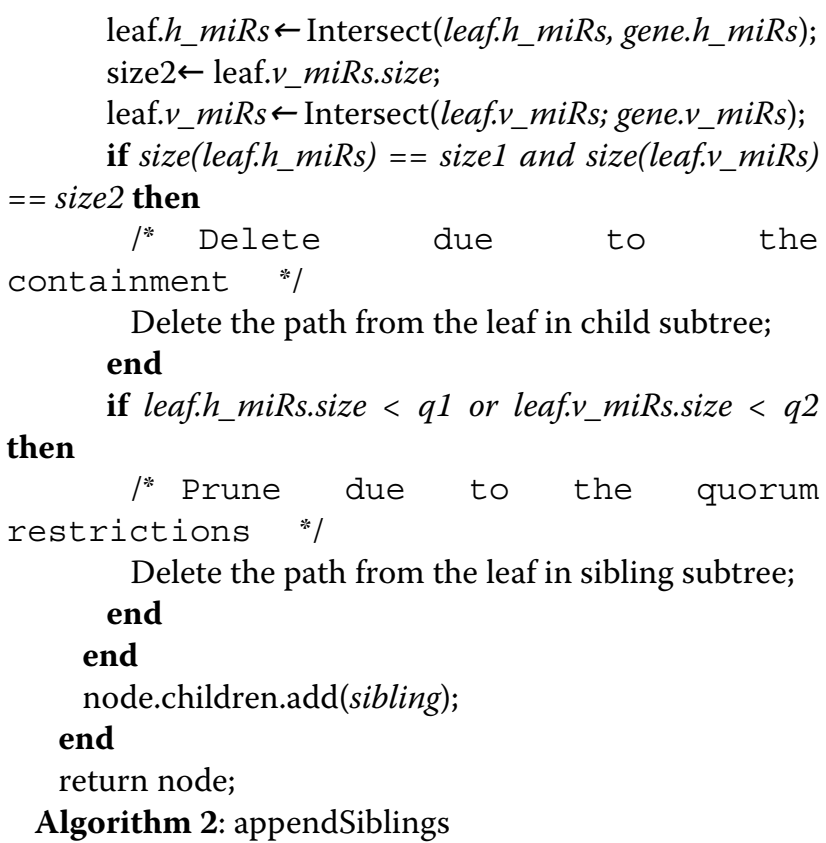

\section{Authors' contributions}

YSA presented the problem and constraint parameters. IVL, KK, and MZU developed the algorithms. IVL contributed to code development and data analysis, and found supporting evidence in the literature. All authors drafted the manuscript, and read and approved the final manuscript.

\section{Acknowledgements}

The authors wish to thank Gary Benson for a helpful discussion on the sampling procedure; Sivan Yogev for code design tips; Tamar Pinhas and Nimrod Milo for pointing the miRecords database. The authors thank the anonymous reviewers for helpful comments.

\section{Author Details}

1Department of Computer Science, Ben-Gurion University, Beer-Sheva 84105 , Israel and 2 Virology and Developmental Genetics/Health Sciences, Ben-Gurion University, Beer-Sheva 84105, Israel

Received: 22 December 2009 Accepted: 13 May 2010

Published: 13 May 2010

\section{References}

1. Engels B, Hutvagner G: Principles and effects of microRNA-mediated post-transcriptional gene regulation. Oncogene 2006, 25(46):6163-6169.

2. Esquela-Kerscher A, Slack F: Oncomirs-microRNAs with a role in cancer. Nature Reviews Cancer 2006, 6(4):259-269.

3. Zhao Y, Srivastava D: A developmental view of microRNA function. Trends in biochemical sciences 2007, 32(4):189-197.

4. Stark A, Brennecke J, Russell R, Cohen S: Identification of Drosophila microRNA targets. PLoS Biol 2003, 1(3):E60.

5. Enright A, John B, Gaul U, Tuschl T, Sander C, Marks D: MicroRNA targets in Drosophila. Genome biology 2004, 5:1-1

6. Lewis B, Shih I, Jones-Rhoades M, Bartel D, Burge C: Prediction of mammalian microRNA targets. Cell 2003, 115(7):787-798.

7. Lewis B, Burge C, Bartel D: Conserved seed pairing, often flanked by adenosines, indicates that thousands of human genes are microRNA targets. Cell 2005, 120:15-20.

8. Kiriakidou M, Nelson P, Kouranov A, Fitziev P, Bouyioukos C, Mourelatos Z, Hatzigeorgiou A: A combined computational-experimental approach predicts human microRNA targets. Genes \& development 2004, 18(10):1165-1178.
9. Krek A, Grun D, Poy M, Wolf R, Rosenberg L, Epstein E, MacMenamin P, da Piedade I, Gunsalus K, Stoffel M, et al.: Combinatorial microRNA target predictions. Nature genetics 2005, 37(5):495-500.

10. Rehmsmeier M, Steffen P, Höchsmann M, Giegerich R: Fast and effective prediction of microRNA/target duplexes. Rna 2004, 10(10):1507-1517.

11. Kim S, Nam J, Rhee J, Lee W, Zhang B: miTarget: microRNA target gene prediction using a support vector machine. BMC bioinformatics 2006, 7:411.

12. Bartel D: MicroRNAs: target recognition and regulatory functions. Cell 2009, 136(2):215-233.

13. Watanabe $Y$, Tomita M, Kanai A: Computational methods for microRNA target prediction. Methods in Enzymology 2007, 427:65-86.

14. Maziere P, Enright A: Prediction of microRNA targets. Drug discovery today 2007, 12(11-12):452-458.

15. Grundhoff A, Sullivan C, Ganem D: A combined computational and microarray-based approach identifies novel microRNAs encoded by human gamma-herpesviruses. Rna 2006, 12(5):733.

16. Scaria V, Hariharan M, Pillai B, Maiti S, Brahmachari S: Host-virus genome interactions: macro roles for microRNAs. Cellular Microbiology 2007, 9(12):2784-2794.

17. Gottwein E, Cullen B: Viral and cellular microRNAs as determinants of viral pathogenesis and immunity. Cell host \& microbe 2008, 3(6):375-387.

18. Barth S, Pfuhl T, Mamiani A, Ehses C, Roemer K, Kremmer E, Jaker C, Hock J, Meister G, Grasser F: Epstein-Barr virus-encoded microRNA miR-BART 2 down-regulates the viral DNA polymerase BALF 5. Nucleic Acids Research 2008, 36(2):666-675.

19. Lecellier C, Dunoyer P, Arar K, Lehmann-Che J, Eyquem S, Himber C, Saib A, Voinnet O: A cellular microRNA mediates antiviral defense in human cells. Science 2005, 308(5721):557-560.

20. Cullen B: Viral and cellular messenger RNA targets of viral microRNAs. Nature 2009, 457(7228):421-425

21. Ghosh Z, Mallick B, Chakrabarti J: Cellular versus viral microRNAs in hostvirus interaction. Nucleic Acids Research 2009, 37(4):1035.

22. Stern-Ginossar N, Elefant N, Zimmermann A, Wolf D, Saleh N, Biton M, Horwitz E, Prokocimer Z, Prichard M, Hahn G, et al:: Host immune system gene targeting by a viral miRNA. Science 2007, 317(5836):376-381.

23. Triboulet R, Mari B, Lin Y, Chable-Bessia C, Bennasser Y, Lebrigand K, Cardinaud B, Maurin T, Barbry P, Baillat V, et al:. Suppression of microRNAsilencing pathway by HIV-1 during virus replication. Science 2007, 315(5818):1579-1582.

24. John B, Enright A, Aravin A, Tuschl T, Sander C, et al.: Human microRNA targets. PLOS Biol 2004, 2(11):e363

25. Watanabe $Y$, Yachie N, Numata K, Saito R, Kanai A, Tomita M: Computational analysis of microRNA targets in Caenorhabditis elegans. Gene 2006, 365:2-10.

26. Lim L, Lau N, Garrett-Engele P, Grimson A, Schelter J, Castle J, Bartel D, Linsley P, Johnson J: Microarray analysis shows that some microRNAs downregulate large numbers of target mRNAs. Nature 2005, 433:769-773.

27. Jarvis M, Borton J, Keech A, Wong J, Britt W, Magun B, Nelson J: Human Cytomegalovirus Attenuates Interleukin-1 \{beta\} and Tumor Necrosis Factor Alpha Proin flammatory Signaling by Inhibition of NF-\{kappa\} B Activation. The Journal of Virology 2006, 80(11):5588-5598.

28. Langland J, Cameron J, Heck M, Jancovich J, Jacobs B: Inhibition of PKR by RNA and DNA viruses. Virus research 2006, 119:100-110.

29. Masucci M: Epstein-Barr virus oncogenesis and the ubiquitinproteasome system. Oncogene 2004, 23(11):2107-2115.

30. Mahalingam S, Karupiah G: Modulation of chemokines by poxvirus infections. Current Opinion in Immunology 2000, 12(4):409-412.

31. Yoon S, De Micheli G: Prediction of regulatory modules comprising microRNAs and target genes. Bioinformatics 2005, 21(2):93-100.

32. Joung J, Hwang K, Nam J, Kim S, Zhang B: Discovery of microRNA-mRNA modules via population-based probabilistic learning. Bioinformatics 2007, 23(9):1141-1147.

33. Tran $D$, Satou $K$, Ho T: Finding microRNA regulatory modules in human genome using rule induction. BMC Bioinformatics 2008, 9(Suppl 12):S5.

34. Madeira S, Oliveira A: Biclustering algorithms for biological data analysis: a survey. IEEE/ACM Transactions on Computational Biology and Bioinformatics 2004, 1:24-45.

35. Kaul A, Stauffer S, Berger C, Pertel T, Schmitt J, Kallis S, Zayas Lopez M, Lohmann V, Luban J, Bartenschlager R: Essential Role of Cyclophilin A for 
Hepatitis C Virus Replication and Virus Production and Possible Link to Polyprotein Cleavage Kinetics. PLoS Pathog 2009, 5(8):e1000546.

36. Perez J, Pham A, Lorini M, Chua M, Steel J, R tenOever B: MicroRNAmediated species-specific attenuation of in uenza A virus. Nature Biotechnology 2009, 27(6):572-576

37. Gusfield D: Algorithms on strings, trees, and sequences: computer science and computational biology Cambridge Univ Pr; 1997.

38. Sagot M: Spelling approximate repeated or common motifs using a suffix tree. Lecture Notes in Computer Science 1998, 1380:374-390.

39. Bernhart S, Tafer H, Mückstein U, Flamm C, Stadler P, Hofacker I: Partition function and base pairing probabilities of RNA heterodimers. Algorithms for Molecular Biology 2006, 1:3.

40. Kertesz M, lovino N, Unnerstall U, Gaul U, Segal E: The role of site accessibility in microRNA target recognition. Nature genetics 2007 39(10):1278-1284.

41. Xiao F, Zuo Z, Cai G, Kang S, Gao X, Li T: miRecords: an integrated resource for microRNA-target interactions. Nucleic Acids Research 2008, 1:1-6.

42. Ensembl [http://www.ensembl.org/biomart/martview/

43. Landgraf P, Rusu M, Sheridan R, Sewer A, lovino N, Aravin A, Pfeffer S, Rice A, Kamphorst A, Landthaler M, et al:: A mammalian microRNA expression atlas based on small RNA library sequencing. Cell 2007, 129(7):1401-1414

44. Rivals I, Personnaz L, Taing L, Potier M: Enrichment or depletion of a GO category within a class of genes: which test? Bioinformatics 2007, 23(4):401-407.

45. Thorley-Lawson D: Epstein-Barr virus: exploiting the immune system. Nat Rev Immunol 2001, 1:75-82.

46. Pratt Z, Kuzembayeva M, Sengupta S, Sugden B: The microRNAs of Epstein-Barr Virus are expressed at dramatically differing levels among cell lines. Virology 2009, 386(2):387-397.

47. miRbase [http://microrna.sanger.ac.uk/

48. Ashburner M, Ball C, Blake J, Botstein D, Butler H, Cherry J, Davis A, Dolinski K, Dwight S, Eppig J, et al:: Gene Ontology: tool for the unification of biology. Nature genetics 2000, 25:25-29.

49. Ragan C, Cloonan N, Grimmond S, Zuker M, Ragan M: TranscriptomeWide Prediction of miRNA Targets in Human and Mouse Using FASTH. PLOS ONE 2009, 4(5):e5745.

50. Mendell J: miRiad roles for the miR-17-92 cluster in development and disease. Cell 2008, 133(2):217-222.

51. O'Donnell K, Wentzel E, Zeller K, Dang C, Mendell J: c-Myc-regulated microRNAs modulate E2F1 expression. Nature 2005, 435(7043):839-843.

52. Xia H, Qi Y, Ng S, Chen X, Chen S, Fang M, Li D, Zhao Y, Ge R, Li G, et al:: MicroRNA- $15 \mathrm{~b}$ regulates cell cycle progression by targeting cyclins in glioma cells. Biochemical and Biophysical Research Communications 2009, 380(2):205-210

53. Cimmino A, Calin G, Fabbri M, Iorio M, Ferracin M, Shimizu M, Wojcik S, Aqeilan R, Zupo S, Dono M, et al:: miR-15 and miR-16 induce apoptosis by targeting BCL2. Proceedings of the National Academy of Sciences 2005, 102(39):13944.

54. Choy E, Siu K, Kok K, Lung R, Tsang C, To K, Kwong D, Tsao S, Jin D: An Epstein-Barr virus-encoded microRNA targets PUMA to promote host cell survival. Journal of Experimental Medicine 2008, 205(11):2551-2560.

55. Han J, Flemington C, Houghton A, Gu Z, Zambetti G, Lutz R, Zhu L, Chittenden T: Expression of bbc3, a pro-apoptotic $\mathrm{BH} 3$-only gene, is regulated by diverse cell death and survival signals. Proceedings of the National Academy of Sciences of the United States of America 2001, 98(20):11318-11323

56. Trigiante G, Lu X: ASPP and cancer. Nature reviews Cancer 2006, 6(3):217-226.

57. Samuels-Lev Y, O'Connor D, Bergamaschi D, Trigiante G, Hsieh J, Zhong S, Campargue I, Naumovski L, Crook T, Lu X: ASPP proteins specifically stimulate the apoptotic function of p53. Molecular cell 2001, 8(4):781-794.

58. Strasser A: The role of BH3-only proteins in the immune system. Nature Reviews Immunology 2005, 5(3):189-200.

59. Mestre-Escorihuela C, Rubio-Moscardo F, Richter J, Siebert R, Climent J, Fresquet V, Beltran E, Agirre X, Marugan I, Marin M, et al:: Homozygous deletions localize novel tumor suppressor genes in B-cell lymphomas. Blood 2007, 109:271-280.
60. Tagawa H, Karnan S, Suzuki R, Matsuo K, Zhang X, Ota A, Morishima Y, Nakamura S, Seto M: Genome-wide array-based CGH for mantle cell lymphoma: identification of homozygous deletions of the proapoptotic gene BIM. Oncogene 2005, 24(8):1348-1358.

61. Clybouw C, Mchichi B, Mouhamad S, Auffredou M, Bourgeade M, Sharma $S$, Leca G, Vazquez A: EBV infection of human B lymphocytes leads to down-regulation of Bim expression: relationship to resistance to apoptosis. The Journal of Immunology 2005, 175(5):2968-2973.

62. Koralov S, Muljo S, Galler G, Krek A, Chakraborty T, Kanellopoulou C, Jensen K, Cobb B, Merkenschlager M, Rajewsky N, et al.: Dicer ablation affects antibody diversity and cell survival in the B lymphocyte lineage. Cell 2008, 132(5):860-874.

63. Ventura A, Young A, Winslow M, Lintault L, Meissner A, Erkeland S, Newman J, Bronson R, Crowley D, Stone J, et al.: Targeted deletion reveals essential and overlapping functions of the miR-17 92 family of miRNA clusters. Cell 2008, 132(5):875-886.

64. Xiao C, Srinivasan L, Calado D, Patterson H, Zhang B, Wang J, Henderson J, Kutok J, Rajewsky K: Lymphoproliferative disease and autoimmunity in mice with elevated miR-17- 92 expression in lymphocytes. Nature immunology 2008, 9(4):405-414.

65. Gressner O, Schilling T, Lorenz K, Schleithoff E, Koch A, SchulzeBergkamen H, Lena A, Candi E, Terrinoni A, Catani M, et al:: TAp63a induces apoptosis by activating signaling via death receptors and mitochondria. The EMBO Journal 2005, 24(13):2458.

66. Flores E, Tsai K, Crowley D, Sengupta S, Yang A, McKeon F, Jacks T: p63 and p73 are required for p53-dependent apoptosis in response to DNA damage. Nature 2002, 416(6880):560-564

67. Serrano $M$, Hannon $G$, Beach $D$, et al:: A new regulatory motif in cell-cycle control causing specific inhibition of cyclin D/CDK4. Nature 1993, 366(6456):704-707.

68. Lukas J, Parry D, Aagaard L, Mann D, Bartkova J, Strauss M, Peters G, Bartek $\mathrm{J}$ : Retinoblastoma-protein-dependent cell-cycle inhibition by the tumour suppressor p16. Nature 1995, 375(6531):503-506.

69. Geradts J, Kratzke R, Niehans G, Lincoln C: Immunohistochemical detection of the cyclin-dependent kinase inhibitor 2 /multiple tumor suppressor gene 1 (CDKN2/MTS1) product p16INK4A in archival human solid tumors: correlation with retinoblastoma protein expression. Cancer Research 1995, 55(24):6006-6011.

70. Reed A, Califano J, Cairns P, Westra W, Jones R, Koch W, Ahrendt S, Eby Y, Sewell D, Nawroz H, et al:: High frequency of p16 (CDKN2/MTS-1/INK4A) inactivation in head and neck squamous cell carcinoma. Cancer Research 1996, 56(16):3630-3633.

71. Sakaguchi M, Fujii Y, Hirabayashi H, Yoon H, Komoto Y, Oue T, Kusafuka T, Okada A, Matsuda $\mathrm{H}$ : Inversely correlated expression of p16 and Rb protein in non-small cell lung cancers: an immunohistochemical study. International Journal of Cancer 1996, 65(4):442-445.

72. Ohtani N, Brennan P, Gaubatz S, Sanij E, Hertzog P, Wolvetang E, Ghysdael J, Rowe M, Hara E: Epstein-Barr virus LMP1 blocks p16INK4a-RB pathway by promoting nuclear export of E2F4/5. Journal of Cell Biology 2003, 162(2):173-183.

73. Lal A, Kim H, Abdelmohsen K, Kuwano Y, Pullmann R Jr, Srikantan S, Subrahmanyam R, Martindale J, Yang X, Ahmed F, et al:: p16INK4a translation suppressed by miR-24. PLoS One 2008, 3(3):e1864.

74. Inomata M, Tagawa H, Guo Y, Kameoka Y, Takahashi N, Sawada K: MicroRNA-17-92 down-regulates expression of distinct targets in different B-cell lymphoma subtypes. Blood 2009, 113(2):396-402.

75. Richter G, Burdach S: ICOS: a new costimulatory ligand/receptor pair and its role in T-cell activion. Onkologie 2004, 27:91-95.

76. Aicher A, Hayden-Ledbetter M, Brady W, Pezzutto A, Richter G, Magaletti D, Buckwalter S, Ledbetter J, Clark E: Characterization of human inducible costimulator ligand expression and function. The Journal of Immunology 2000, 164(9):4689-4696.

77. Mittrucker H, Kursar M, Kohler A, Yanagihara D, Yoshinaga S, Kaufmann S: Inducible costimulator protein controls the protective T cell response against Listeria monocytogenes. The Journal oflmmunology 2002, 169(10):5813-5817.

78. Greenwald R, McAdam A, Woude D Van der, Satoskar A, Sharpe A: Cutting edge: inducible costimulator protein regulates both Th1 and Th2 responses to cutaneous leishmaniasis. The Journal of Immunology 2002, 168(3):991-995. 
79. Bertram E, Tafuri A, Shahinian A, Chan V, Hunziker L, Recher M, Ohashi P, Mak T, Watts T: Role of ICOS versus CD28 in antiviral immunity. European journal of immunology 2002, 32(12):3376-3385.

80. Aho A, Hopcroft J, Ullman J: Data structures and algorithms AddisonWesley Longman Publishing Co., Inc. Boston, MA, USA; 1983

81. Cullen B: Viruses and microRNAs. Nature genetics 2006, 38:25-30.

doi: 10.1186/1471-2105-11-249

Cite this article as: Veksler-Lublinsky et al., Gene bi-targeting by viral and human miRNAs BMC Bioinformatics 2010, 11:249

Submit your next manuscript to BioMed Central and take full advantage of:

- Convenient online submission

- Thorough peer review

- No space constraints or color figure charges

- Immediate publication on acceptance

- Inclusion in PubMed, CAS, Scopus and Google Scholar

- Research which is freely available for redistribution

Submit your manuscript at www.biomedcentral.com/submit
() BioMed Central 